\title{
Aerodynamic Characteristics of Fairing Separation at Initial Opening Angle
}

\author{
Xuechang Zhu \\ School of Aeronautics \\ Northwestern Polytechnic University \\ Xi'an, China
}

\author{
Xiaojing $\mathrm{Yu}$ \\ School of Power and Energy \\ Northwester Polytechnic University \\ Xi'an, China
}

\author{
Hong Yan \\ School of Power and Energy \\ Northwester Polytechnic University \\ Xi'an, China
}

\begin{abstract}
Numerical analyses were carried out to investigate the aerodynamic characteristics of clamshell fairing separation at Mach 5 in low altitude atmosphere. A three dimensional numerical model was set up. The flow fields of the closed fairing and condition at the initial opening angle were simulated. Results showed that the complicated shock waves formed in the multibody system, and the interference of shock waves greatly changed the aerodynamic force acting on the fairing and flying vehicle. The time history of the force was obtained. The minimum initial angular velocity to avoid the fairing re-closing could be predicted based on the present study.
\end{abstract}

Keywords-clamshell fairing; separation; aerodynamic force; initial angular velocity

\section{INTRODUCTION}

The launch vehicle is protected by a fairing during the process of setting the payload into an orbit. The fairing shields the vehicle from aerodynamic loads, heating and vibration, etc. When flying to a certain altitude, the faring should be jettisoned to meet the demands of the flying requirement. Usually, the separation process takes place above the altitude of $80 \mathrm{~km}$. While in recent years, there is an increasing demand of expanding the capability of launch vehicles, which leads to fairing separation in low altitude atmosphere [1, 2]. In dense atmosphere, the aerodynamic force is of great influence on the movement of the fairing because of the high dynamic pressure. The trajectory of the fairing is affected by the complicated flow phenomena, such as shock waves, turbulent flow, etc. Therefore, the aerodynamic characteristics of the fairing are critical to the safety of the vehicle.

The clamshell fairing is one of the most commonly used types. One fairing composes of two halves, and each half of the fairing is connected to hinges, which locate at the bottom of the fairing and joint the fairing with the upper stage. When receive the separation order, the clamp device is unlocked. Under the effects of initial force, such as spring force or explosive force, the halves open to a small initial angle , and then rotate around the hinges under the effects of aerodynamic force; when reach the pre-set detach angle, the fairing separate from the vehicle and fly downstream. The process is shown in figure 1.

Two issues need addressing to ensure the safe separation. First, when the fairing halves open at initial small angle, they should not re-close. At this initial opening angle, the faring and the central vehicle are closed to each other and have mutual influence. The flow field is complicated and the movement of the faring is determined by the initial angular velocity and aerodynamic force. Second, when the fairing detaches from the vehicle, they should not collide with the vehicle. In this freeflight stage, the trajectory of the fairing is mainly depended on the aerodynamic force.
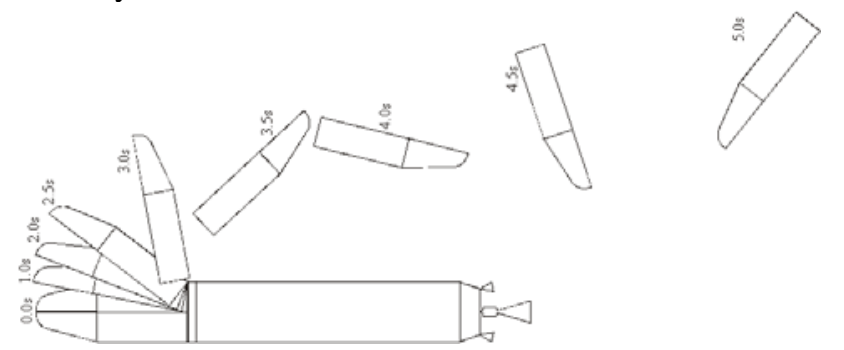

Figure 1. Schematic view of chamshell fairing separation[3]

In the last few decades, some of the investigations were carried out to study the multi-body separation process. Constrained motion equation method was adopted by Robert et al. [4], Tartabini et al. [5], and Oh et al [6]. The separation system was molded as two-rigid body and a joint, and the dynamic motion equations were deduced. The movement of the fairing before fully detached from vehicle could be obtained. The minimum permissible relative velocity of the fairing and the spring force to initiate the opening were present. The multibody dynamic simulation method was adopted for the elastic fairing separation [7]. The unsteady aerodynamic load was added to the motion and structure analysis. The dynamic mesh method was adopted by Wang et al. [8] to simulate the clamshell fairing separation process. The "centroid backward rule" was proposed according to the fairing trajectory simulation to ensure the safe separation.

In this paper, we focused on the initial stage of small opening angle of clamshell fairing separation. The mutual influence of the faring and the central body was revealed, and the aerodynamic characteristics were analyzed.

\section{GEOMETRY MODEL AND NUMERICAL METHOD}

A scaled geometry was modeled as shown in figure 2 . The separation system composed of a central body and two halves of fairings. The origin of the coordinate lies on the stagnation point of the central body, with the $\mathrm{x}$ axis aligned with the axis 
of the central body. The $x-y$ plane is cut through the center of central body. The hinge center was located at the bottom of the fairings in the $\mathrm{y}=0$ plane, and its axis paralleled with the $\mathrm{y}$ axis. The fairings rotate around the hinge center. The length to diameter ratio of the fairing is $L / D=3$, where $L$ is the total length of the fairing and $\mathrm{D}$ is the diameter of the cylindrical section of the fairing. The fairing separation altitude was $25 \mathrm{~km}$ at Mach=5.

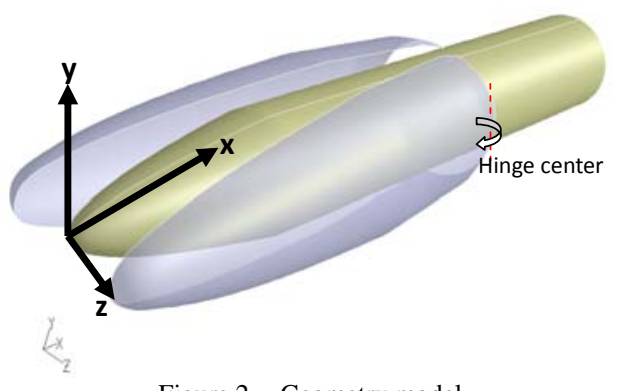

Figure 2. Geometry model

A three dimensional numerical model was establish with the flow solver FLUENT. Because the geometry is symmetric about $\mathrm{z}=0$ plane, only half of the flow field was simulated. The second order AUSM scheme was adopted for the convective flux term. And the turbulent model was sst $-\kappa \omega$ model.

\section{RESULTS AND DISCUSSION}

\section{A. Fairing closed}

Firstly, the flow field with fairing closed was simulated. The numerical schlieren image was shown in figure 3. It could be seen that a bow shock formed around the fairing. The wave drag became a big portion of the total drag in hypersonic flow. The temperature contour in the $\mathrm{z}=0$ and $\mathrm{y}=0$ plane and pressure contour in fairing surface were shown in figure 4. It was observed that a high temperature and high pressure region was formed in the head of the fairing. The fairing protected the central body from aerodynamic force and heating when closed.

Take the cylinder cross section area of the fairing as the reference area, the drag coefficient of the half fairing was $C_{x, \text { fairing }}=206$ and the aerodynamic coefficient in $\mathrm{z}$ direction was $C_{z \text {, fairing }}=-502$. It could be inferred that the aerodynamic force in $\mathrm{z}$ direction $f_{z \text {,fairing }}$ pressed the two halves closed. To make the fairing opened, the momentum of the initial force to the hinge center should be greater than the momentum of $f_{z \text {, fairing }}$, and had a reverse direction. According to the relation, the minimum initial force could be determined.
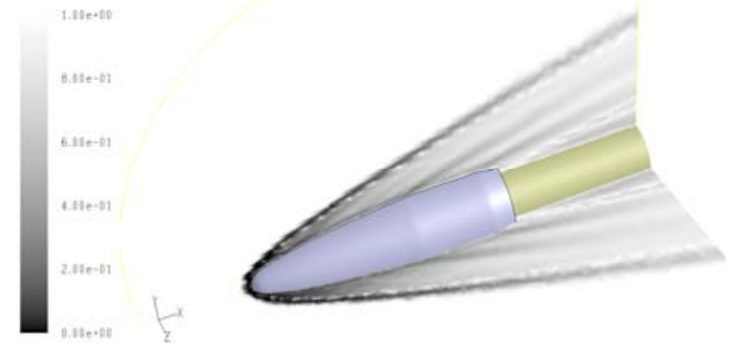

Figure 3. Numerical schlieren image in fairing closed condition

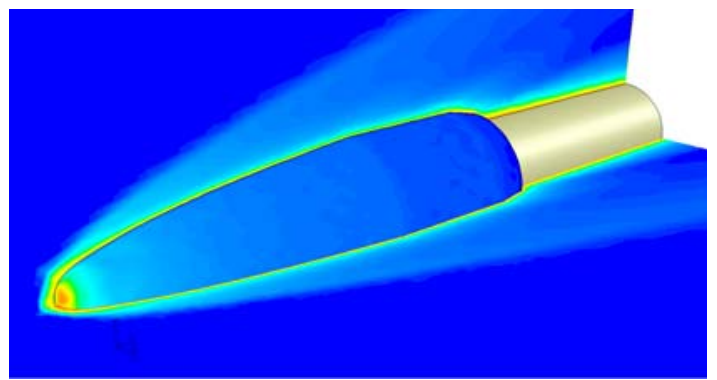

Figure 4. Temperature contour in $\mathrm{z}=0$ and $\mathrm{y}=0$ plane; pressure contour in fairing surface

\section{B. Initial opening angle}

When the fairing opened to a small angle under the effects of the initial force caused by springs or explosives, the flow began filling the internal zone of the fairing. The pressure on the inner and outer surface of the fairing would not reach balance instantaneously. The aerodynamic force changed with the filling process of the flow, and thus influenced the movement of the fairing. To explore the characteristics of the aerodynamic force, a transient simulation should be performed. Because the fairing jettison duration was on the order of a few seconds, while the flow filling process was a few milliseconds. The displacement of the fairing was negligible during a short period of time. Therefore, we could assume that, during the short flow filling process, the faring was fixed at a certain opening angle although the aerodynamic force was changing. In this case, the initial opening angle was set to 2 degree.

The time history of aerodynamic coefficient of the central body and fairing was plotted in figure 5. It was shown that, before 0.5 millisecond, the change of the aerodynamic force was large. The drag of the body was maximum at start, and decreased with time. The force of the fairing in the negative $\mathrm{z}$ direction was maximum at the start. That is to say, the angular acceleration of the fairing was negative at the start. If the initial angular velocity was small, the fairing has the possibility of reclosing under the effects of aerodynamic force. If the mass of the fairing was known, the angular acceleration and angular velocity could be calculated. Therefore, the minimal initial angular velocity to avoid re-closing could be predicted. After 0.5 millisecond, the drag force of the central body approached a certain value, while the force acting on the fairing in $\mathrm{z}$ direction began to be positive. It means that, the aerodynamic force tended to accelerate the separation process at the later stage. 


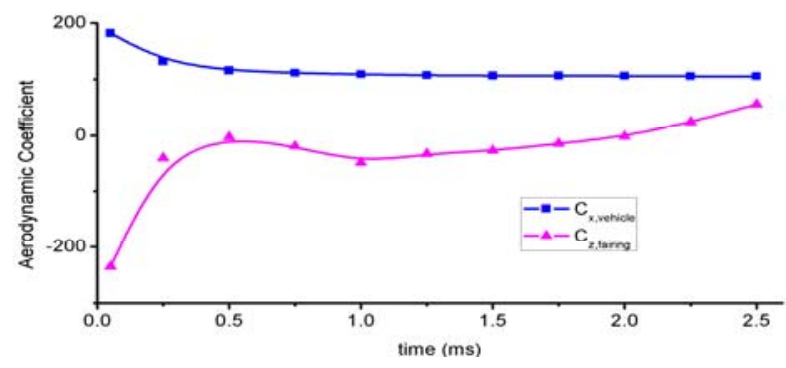

Figure 5. Time histrory of drag coefficnet of central body $C_{x, v e h i c l e}$ and $\mathrm{z}$ direction force coefficient $C_{z \text {, fairing }}$

The flow field at three critical moments was shown in figure $6 \sim$ figure 8 . At $t \approx 0.05 \mathrm{~ms}$ (figure 6 ), the shock wave formed by the fairing impact at the head of the central body, and formed a high pressure region, which increased the drag of the body. A separation vortex was formed in the concaved inner surface of fairing. The shock wave formed by the body impinged at the separation region and deflected. This shock wave increased the inner surface pressure of the fairing, and was benefit for the rotation of the fairing in the opening direction. Although it was still comparatively large, the magnitude of $f_{z \text {, fairing }}$ has already greatly reduced in the negative $\mathrm{z}$ direction when compared with the fairing closed condition. At $t \approx 0.5 \mathrm{~ms}$ (figure 7 ), with the flow filling into the space between the central body and fairing, the shock interference patter changed a lot. The shock wave of the body impact at the inner surface of the faring and reflected, and the reflected wave impact the central body surface. The multiple reflected waves increased the pressure of the faring inner surface, and the pressure difference between the inner and outer surface was reduce. At $t \approx 1.5 \mathrm{~ms}$ (figure 8 ), the distribution of the waves turned to be comparatively stable, and the aerodynamic force approached a certain value.
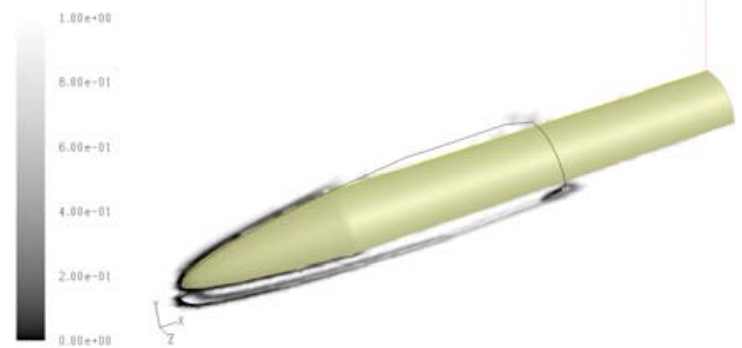

a) Numerical schlieren image

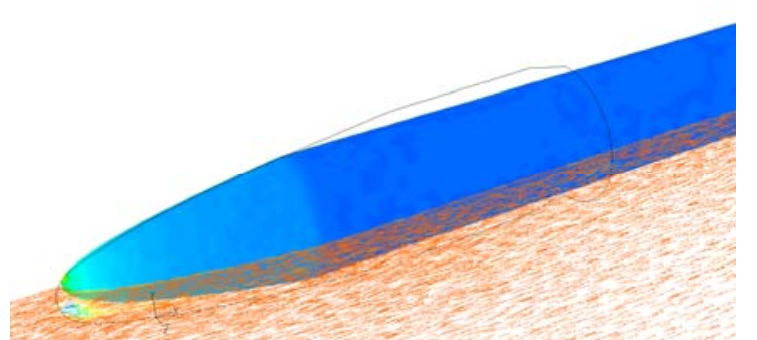

b) Velocity vector at $y=0$ plane and surface pressure on the central body

Figure 6. $\mathrm{t}=0.05 \mathrm{~ms}$

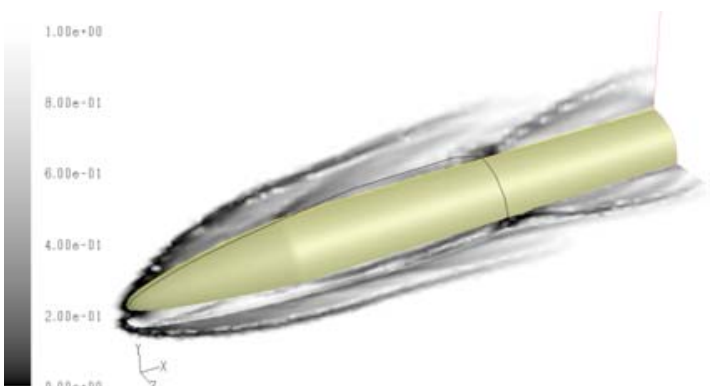

a) Numerical schlieren image

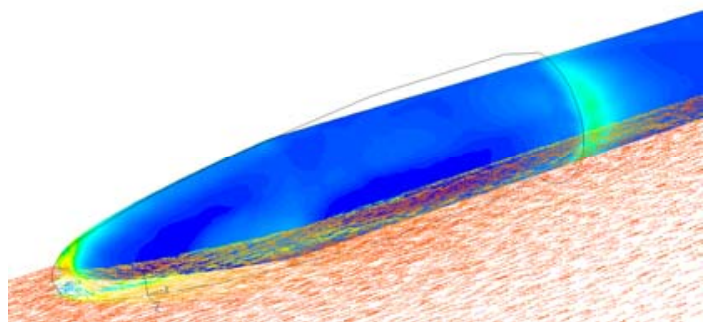

b) Velocity vector at $y=0$ plane and surface pressure on the central body Figure 7. $t=0.5 \mathrm{~ms}$

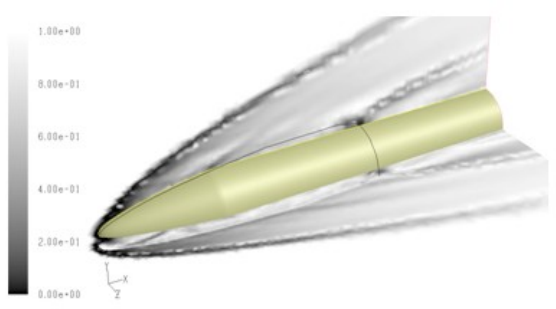

a) Numerical schlieren image

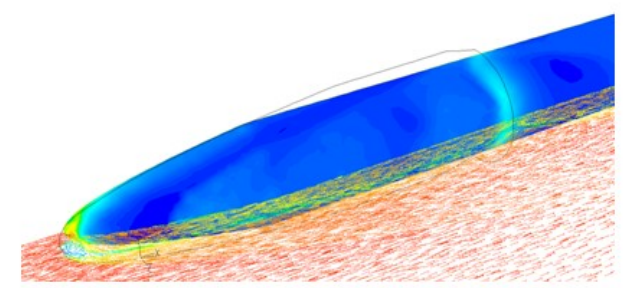

b) Velocity vector at $y=0$ plane and surface pressure on the central body

Figure $8 . \mathrm{t}=1.5 \mathrm{~ms}$

\section{CONCLUSIONS}

Numerical simulations were carried out to investigate the aerodynamic characteristics of clamshell fairing separation at Mach 5 in low altitude atmosphere. The flow field of the closed fairing and the condition with 2 degree opening were simulated. The transient process of flow filling into the fairing at the initial opening angle of 2 degree was studied. The results showed that the flow field between the body and fairing was complicated, including a series of reflected waves. The interference shock waves increased the inner surface pressure of the fairing, which was of great benefit for the separation. The time history of the drag coefficient on the central body and the aerodynamic coefficient on the fairing was obtained. The minimum initial 
angular velocity to avoid fairing re-closing could be predicted based on the analysis.

\section{REFERENCES}

[1] A.V. Beloshitsky, Yu.I. Grigoriev, and A. A. Dyadkin, et al. "Aerodynamic effect on spacecrafts during head firing jettison in dense atmosphere layers," Proc. $4^{\text {th }}$ Europ Symp. Aerothermodynamics for Space Applications,15-18 Oct. 2001.

[2] J.Liu, W.Wang, Z.Guo, and B. Liu, "Numerical simulation of the detaching process for rocket mantles in low altitude,” J. of Ballistics, vol.18, no.3, Sep. 2006, pp.34-38.

[3] Long March 2c User’s Manual

[4] C. Robert and J. Saunders, "Equations of motion of a constrained split fairing system during opening," J. of Spacecraft, 1972, pp.55-57
[5] P.V.Tartabini, C.M.Roithmayr, et al. Elissa, "Modeling multibody stage separation dynamics using constraint force equation methodology,” J. of Spacecraft and Rockets, vol.48,no.4,2011, pp.573-583

[6] C.S.Oh, B.C. Sun et al., "Payload fairing separation analysis using contraint force equation," International Conference on Control, Automation and System 2010.

[7] X.W. Zhang, Y.R. Wang, et al., "Fluid-structure interaction method on numerical simulation of elastic fairing separation,” J.of Beijing University of Aeronautics and Astronautics, vol. 35, nol8, 2009, pp. 976-979,

[8] W.Wang, "Numerical simulation technique research and experiment verification for unsteady multi-body flow field involving relative movement,”, PHD dissertation, National University of Defense Technology, 2008. 